\title{
La microfluidisation comme procédé d'homogénéisation d'une boisson à base de matière grasse laitière
}

\author{
P. Paquin et J. Giasson
}

Université Laval, Faculté des sciences de l'agriculture et l'alimentation, groupe de recherche Stela, Québec, Canada

(reçu le 21 décembre 1988, accepté le 18 avril 1989)

\begin{abstract}
Résumé - L'homogénéisation des boissons alcoolisées à base de matière grasse laitière est un procédé permettant d'allonger le temps d'entreposage de ces produits. La microfluidisation est une nouvelle technologie favorisant une réduction significative de la taille des globules de matière grasse d'une boisson alcoolisée et elle a été comparée à l'homogénéisation conventionnelle. Nos résultats démontrent que ce procédé permet une meilleure stabilité du produit par une réduction de la taille des globules de gras de même que l'obtention d'un profil de distribution plus étroit de ces particules. Le diamètre moyen obtenu par analyse de diffusion de la lumière est de 0,1 contre $0,2 \mu \mathrm{m}$ pour le procédé traditionnel. Ce procédé s'avère très efficace pour les émulsions liquides à des fins de conservation prolongée.
\end{abstract}

microfluidisation - homogénéisation - émulsion liquide - longue stabilité - laser light scattering (diffusion de la lumière) - boisson alcoolisée

Summary - Microfluidization as an homogenization process for cream liqueur. The homogenization of cream liqueur is a process that lengthens shelf-life of the product. Microfluidization, a novel technology, allows a significant reduction of the fat particule size in a cream liqueur and it has been compared to conventional homogenization. Our results show that this new technology yields a product with a higher stability due to the reduction of the average diameter of the fat particules. The diameter of the particles analyzed by laser light scattering is $0.1 \mu \mathrm{m}$ for the microfluidization compared to $0.2 \mu \mathrm{m}$ for conventional homogenization. Microfluidization shows great potential for liquid emulsions requiring a long shelf-life.

microfluidization - homogenization - liquid emulsion - long shelf lige - laser light scattering - cream liqueur 


\section{INTRODUCTION}

L'homogénéisation de certaines émulsions alimentaires, et plus précisément l'homogénéisation des produits laitiers, est un procédé employé industriellement au Canada depuis 1927 (McCormick, 1968). Cette technologie, développée au début des années 1900 , consiste à faire passer, sous pression, un liquide par une fente étroite. Dans le cas d'une émulsion laitière, la fente utilisée est normalement de dimension quelque peu plus large que le diamètre réel des globules gras. La forte pression exercée sur le liquide développe des forces de cisaillement, de cavitation et de micro-turbulence, qui agissent sur les globules de matière grasse en provoquant la réduction de leur taille.

Pour les produits laitiers, cette technologie permet de réduire le diamètre des globules gras, qui varie de 2 à $6 \mu \mathrm{m}$ dans le lait cru (Kessler, 1981), à un diamètre moyen de $0,7 \mu \mathrm{m}$. Le but visé dans cette opération est de ralentir la remontée de la matière grasse à la surface du produit. En effet, selon la loi de Stokes (Walstra \& Jenness, 1984), lorsque des particules en suspension dans une phase liquide ont une densité plus faible que celle-ci, la vitesse de remontée de ces particules est directement proportionnelle au carré de leur diamètre et inversement proportionnelle à la viscosité du liquide.

L'évolution du domaine laitier au cours des dernières années a cependant créé un besoin de recherche et développement de technologies plus performantes afin d'obtenir des émulsions dont la durée de conservation est encore supérieure. Cela est illustré par les produits de type UHT qui possèdent un temps de conservation de 6 mois à 1 an. On observe également un développement des marchés d'exportation sous toutes formes (poudres, concentrés et liquides).
L'arrivée récente d'une nouvelle technologie d'homogénéisation pourrait permettre une meilleure qualité de ces produits à conservation prolongée. La microfluidisation, qui est apparue dans les années 80 (Cook \& Lagace, 1985), permet la formation de microémulsions. Cette technologie fut développée afin de préparer des dispersions liquide-liquide ou liquide-solide dans des domaines de la médecine, de la pharmacologie (Mayhew et al., 1984) et des cosmétiques (Chandonnet et al., 1985; Siliciano, 1985).

Les résultats de l'étude présentée ici découlent de l'application de cette technologie à la formulation des produits laitiers de longue conservation. D'après la littérature, les digestifs à base de crème alcoolisée ont une stabilité prolongée lorsque le diamètre des particules de matière grasse du produit est petit (Muir \& Banks, 1986 a et b). En s'appuyant sur les résultats de ces auteurs, nous avons comparé la microfluidisation au procédé d'homogénéisation conventionnel.

\section{MATÉRIEL ET MÉTHODES}

\section{Principe de la technique}

L'appareil utilisé est un microfluidisateur TM 110 , d'un débit maximum de $36 \mathrm{l} / \mathrm{h}$ et permettant des pressions de 0 à $76 \mathrm{MPa}$ (Microfluidic Corporation, Boston, Massachusetts, USA). La compagnie nous a fourni cet appareil de type laboratoire afin d'effectuer des essais sur des émulsions alimentaires. Le principe de la microfluidisation consiste à diviser le liquide d'entrée en 2 feuillets de liquide identiques que l'on appelle microcanaux. Ces microcanaux sont, par la suite, dirigés à un endroit spécifique de l'appareil appelé chambre de réaction. A cet endroit, les 2 jets de liquide viennent en collision à un angle de $180^{\circ}$ (Fig. 1). Lors de cette opération, des forces de cisaillement, de cavitation et de turbulence se développent. Ces forces agis- 


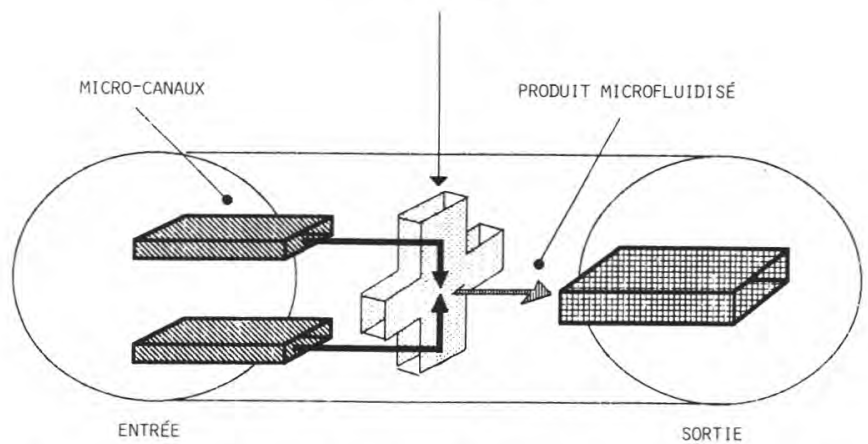

Fig. 1. Schéma de la chambre de réaction du microfluidisateur (d'après Microfluidics Corp., 1985).

sent sur les globules de gras afin de favoriser la réduction de leur taille. La collision des liquides, de même que la géométrie de la chambre de réaction, permettent d'utiliser de très hautes pressions. La pression interne est relâchée dans l'espace de $10^{-6} \mathrm{~s}$ à la sortie de la chambre de réaction, permettant ainsi un procédé constant et peu dommageable pour le produit, ce qui donne des émulsions plus homogènes.

La compagnie Microfluidic Corporation fabrique également des appareils semi-industriels permettant des débits allant jusqu'à $13000 \mathrm{l} / \mathrm{h}$.

\section{Composition de l'émulsion}

La crème alcoolisée utilisée pour ce travail de recherche est une formulation de base comparable à cell que l'on retrouve dans la littérature (Banks et al., 1981a et b, 1983, 1985). C'est une émulsion contenant $13,0 \%$ de matière grasse dispersée dans une phase aqueuse contenant $12,6 \%$ d'alcool. La composition détaillée du produit est présentée au Tableau I et le procédé de fabrication est décrit à la Figure 2.

Tableau I. Composition d'une crème liqueur

Formulation (ingrédients) $\%(p / p)$

Matière grasse (crème $50 \% \mathrm{mg}$ )

13,00

Sucre (sucrose $70 \%$ )

23,40

Stabilisant (caséinate de sodium)

Emulsifiant (monostéarate de glycérol)

0,25

Citrate de sodium

0,38

Eau

47,37

Alcool (éthanol 40\%)

12,60 




Fig. 2. Procédé de fabrication de la crème alcoolisée.

\section{Evaluation analytique}

Profil de distribution du diamètre des globules gras

Afin d'évaluer le diamètre des globules gras, nous utilisons la technique de diffusion de la lumière à partir d'une source de lumière laser (Laser Light Scattering). L'appareil utilisé est un Nicomp model 370 Submicron Particle Sizer (Pacific Scientific, Santa Barbara, Ca., USA). Le Nicomp détermine la dimension des particules de l'émulsion d'après les fluctuations de l'intensité de la lumière diffusée (dynamic light scattering), causées par le mouvement brownien des particules. L'échantillon injecté est dilué automatiquement et cette dilution est toujours rapportée au maximum de l'intensité de dispersion et ce indépendamment de la concentration de départ.

\section{Déstabilisation par centrifugation}

La méthode utilisée est une modification de la méthode de Maxcy \& Sommer (1953); elle se veut un test d'accélération dans le temps de la remontée des globules gras, de façon à évaluer la qualité de l'émulsion.

Cent cinquante $\mathrm{ml}$ d'émulsion sont placés dans les flacons de $250 \mathrm{ml}$.
Les échantillons sont centrifugés à une vitesse de $16319 \mathrm{~g}$ pendant $30 \mathrm{~min}$ à température ambiante (Sorvall RC-5B, Rotor \# GSA $14,61 \mathrm{~cm}$, DuPont Instruments, USA.

A l'aide d'une pipette Pasteur reliée à un tube à succion, on recueille la portion du fond de l'échantillon, soit $50 \mathrm{ml}(1 / 3)$, et ce en évitant toute agitation.

Le contenu en matière grasse de cette portion de l'échantillon est déterminé par analyse à l'infrarouge, de même que le contenu en matière grasse de l'échantillon de départ avant centrifugation (Milko-Scan 104 Foss Electrik, Denmark).

L'indice de stabilité est alors exprimé en pourcentage de la façon suivante :

matière grasse de l'échantillon centrifugé $X 100$

matière grasse de l'échantillon de départ

\section{Evaluation de la stabilité à l'entreposage}

Nous évaluons également la stabilité des émulsions de crème alcoolisées par l'entreposage d'échantillons à des températures élevées. Cette pratique est utilisée en industrie afin d'accélérer le vieillissement des émulsions.

Les échantillons de $150 \mathrm{ml}$ sont placés à une température de $38^{\circ} \mathrm{C}$ pendant 6 mois. Par 
la suite, $50 \mathrm{ml}$ de la phase inférieure de l'émulsion est pipettée par succion, de la même manière que pour le test de centrifugation et l'indice de stabilité est aussi déterminé par la même méthode.

\section{RÉSULTATS ET DISCUSSION}

\section{Microfluidisation versus homogénéisa- tion}

La Figure 3 représente l'analyse par diffusion de lumière pour une crème liqueur microfluidisée comparée à un produit commercial homogénéisé. Le profil de distribution des particules de matière grasse obtenu nous révèle essentiellement 2 choses : la courbe $M$ qui représente l'échantillon microfluidisé (29 $\mathrm{MPa}$ ) pos-

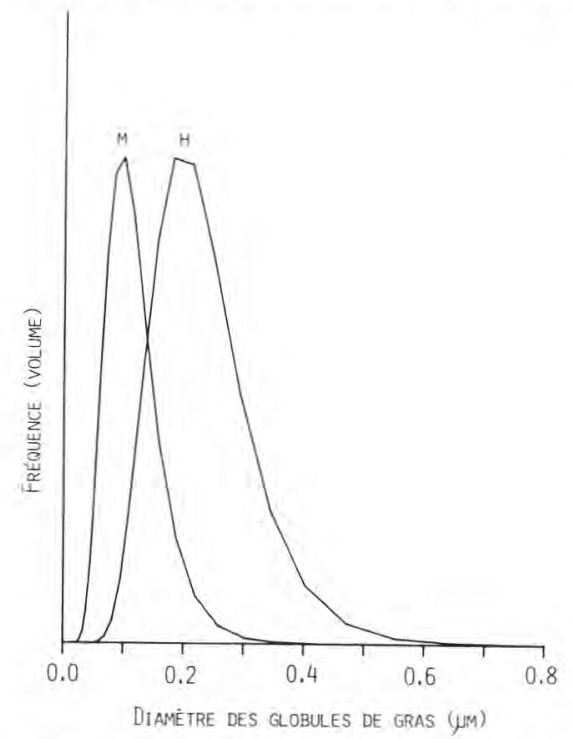

Fig. 3. Analyse du profil de distribution du diamètre des globules gras pour une crème alcoolisée microfluidisée à $29 \mathrm{MPa}(\mathrm{M})$ comparée à un produit commercial ayant subi une homogénéisation classique à $30 \mathrm{MPa}(\mathrm{H})$. sède un diamètre moyen de $0,13 \mu \mathrm{m}$ avec des valeurs s'échelonnant de 0,04 à 0,40 $\mu \mathrm{m}$, comparativement à l'échantillon commercial homogénéisé ( $30 \mathrm{MPa}$ ) qui possède un diamètre moyen de $0,21 \mu \mathrm{m}$ et des diamètres s'échelonnant de 0,06 à $0,65 \mu \mathrm{m}$. Ces résultats nous permettent d'observer que la microfluidisation, non seulement favorise l'obtention de particules de matière grasse de diamètre plus petit, mais fournit un système polydispersé possédant une distribution plus étroite $(0,04$ à $0,40 \mu \mathrm{m}$ comparé à 0,06 à 0,65 $\mu \mathrm{m})$. Cela est d'autant plus significatif que l'on s'accorde à dire que c'est surtout la faible proportion de particules possédant un plus gros diamètre qui va causer l'instabilité d'une émulsion. L'équation de Stokes nous permet de confirmer cette affirmation puisque :

$$
\frac{V=D^{2}\left(\rho_{2}-\rho_{1}\right) g}{18 \eta}
$$

où $V$ est la vitesse de migration proportionnelle au carré du diamètre (D) des particules, les autres facteurs de l'équation étant constants pour un même type d'émulsion comme dans notre cas (Walstra \& Jenness, 1984). Ces facteurs sont $\rho_{1}$ et $\rho_{2}$, les densités respectives de la matière grasse et de la phase aqueuse, $\mathrm{g}$ la force gravitationnelle et $\eta$ la viscosité dynamique du milieu.

La Figure 4 représente une série de courbes de distribution des diamètres de globules gras pour des produits microfluidisés à différentes pressions. Les résultats démontrent qu'il est possible de faire varier le diamètre moyen des particules en fonction de la pression. On observe un déplacement progressif du diamètre moyen et de la courbe de distribution vers des valeurs plus faibles, et ce en fonction d'une pression de microfluidisation plus élevée (de 29 à $48 \mathrm{MPa}$ ). 


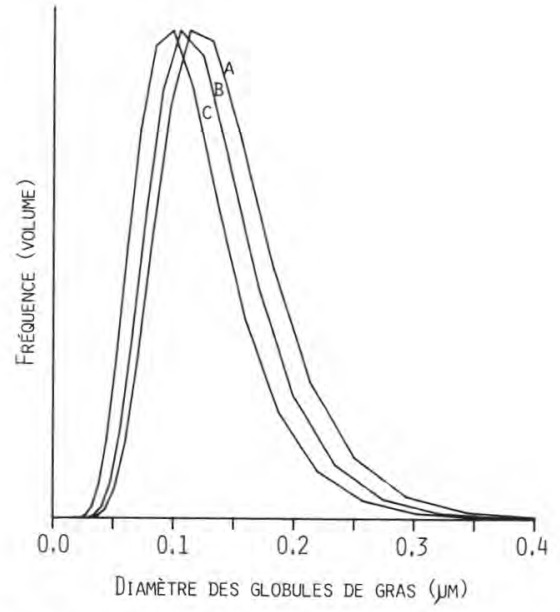

Fig. 4. Analyse du profil de distribution du diamètre des globules gras pour des crèmes alcoolisées ayant subi différentes pressions de microfluidisation, soit à $29 \mathrm{MPa}(\mathrm{A}), 39 \mathrm{MPa}(\mathrm{B})$ et 48 $\mathrm{MPa}(\mathrm{C})$.

Cette analyse par diffusion de lumière nous permet de constater un effet important de la microfluidisation sur les particules présentes dans une émulsion comme les boissons alcoolisées à base de crème. Les résultats obtenus démontrent que, sur une base comparative et non sur une valeur absolue, il est évident que la microfluidisation produit des émulsions plus fines que l'homogénéisation conventionnelle. Les valeurs obtenues par diffusion de lumière ne peuvent être considérées comme des valeurs absolues à cause de la présence de micelles de caséines dans l'émulsion. Les micelles de caséines du lait possèdent un diamètre moyen de l'ordre de $0,13 \mu \mathrm{m}$ (Walstra \& Jenness, 1984), ce qui interfère avec la mesure des particules de gras. Cependant, on peut considérer cette interférence comme négligeable puisqu'elle est présente tant dans l'émulsion microfluidisée que celle homogénéisée.

Les figures 3 et 4 démontrent clairement que la microfluidisation est une technologie de pointe plus performante que l'homogénéisation pour la production d'émulsions fines.

\section{Stabilité des émulsions microfluidisées}

Le Tableau II présente les résultats obtenus pour les tests de stabilité par centrifugation et traitement thermique des échantillons microfluidisés et de l'échantillon commercial. L'étape de microfluidisation à différentes pressions est effectuée sur le produit fini après l'ajout d'alcool. Les résul-

Tableau II. Effet de la pression de microfluidisation de la crème liqueur sur la stabilité du produit.

\begin{tabular}{lccc}
\hline $\begin{array}{c}\text { Pression de } \\
\text { microfluidisation } \\
(\mathrm{MPa})\end{array}$ & $\begin{array}{c}\text { Diamètre moyen des } \\
\text { globules gras } \\
(\mu \mathrm{m})\end{array}$ & $\begin{array}{c}\text { Indice de } \\
\text { stabilité }(\%) \\
\text { Centr. }\end{array}$ & Tr. ther. $^{* \star}$ \\
\hline 29 & 0,13 & 83,6 & 76,6 \\
39 & 0,12 & 87,6 & 92,8 \\
48 & 0,10 & 93,1 & 86,3 \\
30 (commercial) & 0,21 & 84,8 & 93,4 \\
\hline
\end{tabular}

\footnotetext{
* Centrifugation. ** Traitement thermique $\left(38^{\circ} \mathrm{C}\right)$.
} 
tats du test de centrifugation donnent une bonne corrélation entre la réduction du diamètre moyen et l'accroissement de stabilité de $84 \%$ à $93 \%$. Cependant, les résultats obtenus pour le test par traitement thermique sont plus incohérents. Cela peut être associé à l'augmentation de la fluidité de la membrane stabilisatrice de ces globules à une température plus élevée, ce qui a pour effet de diminuer sa résistance mécanique.

Lorsque l'on compare les résultats obtenus pour les échantillons microfluidisés à ceux de l'échantillon commercial, on observe que dans le cas du test accéléré par centrifugation on peut augmenter l'indice de stabilité de $10 \%$, ce qui n'est pas le cas pour le traitement thermique. Pour obtenir une idée plus exacte de la stabilité des échantillons, ceux-ci seront analysés après conservation de un an à température ambiante.

\section{CONCLUSION}

Les résultats obtenus dans cette étude nous ont permis de démontrer l'efficacité d'une technologie comme la microfluidisation, surtout si on observe nos résultats concernant la mesure des diamètres moyens ainsi que le profil de distribution des diamètres des particules de matière grasse dans l'émulsion microfluidisée. Ces résultats démontrent également l'intérêt industriel de la technique pour la fabrication de digestifs à base de crème alcoolisée. La microfluidisation est une technologie tellement efficace quant à la réduction de taille des globules gras qu'un seul traitement en fin de formulation est aussi efficace que la double homogénéisation utilisée de façon conventionnelle.

La microfluidisation est une technologie de pointe qui représente un grand potentiel pour les émulsions liquides. II y a certainement un avantage à utiliser cette technologie dans différents types d'émulsions alimentaires.

Les travaux de recherche sur la formation de microémulsion à l'aide de cette technologie sont présentement en cours.

\section{REMERCIEMENTS}

Nous présentons nos remerciements au centre de recherches alimentaires de St-Hyacinthe (Crash, Agriculture Canada) pour son assistance financière dans ce projet, ainsi que le ministère de l'Agriculture, des Pêcheries et de l'Alimentation du Québec.

\section{RÉFÉRENCES}

Banks W., Muir D.D. \& Wilson A.G. (1981a) Extension of the shelf life of cream-based liqueurs at high ambient temperatures. J. Food Technol. $16,587-595$

Banks W., Muir D.D. \& Wilson A.G. (1981b) The formulation of cream-based liqueurs. Milk Ind. 83, 16-18

Banks W., Muir D.D. \& Wilson A.G. (1983) Stabilization of alcoholic beverages by sodium caseinate. In : Physico-Chemical Aspects of Dehydrated Protein-Rich Milk Products. Proc. IDF Symp., Helsingor, Denmark, pp. 331-338

Banks W. \& Muir D.D. (1985) Effect of alcohol content on emulsion stability of cream liqueurs. Food Chem. 18, 139-152

Chandonnet S., Korstvedt H. \& Siciliano A.A. (1985) Preparation of microemulsions by microfluidization. Soap, Cosmetics, Chemical Specialties.

Cook E.J. \& Lagace A.P. (1985) Apparatus for forming emulsions. United States Patent 4, 533, 254

Kessler H.G. (1981) Emulsifying. Homogenizing. In : Food Engineering and Dairy Technology. Verlag A. Kessler, Germany, pp. 119-138 
Maxcy R.B. \& Sommer H.H. (1953) Fat separation in evaporated milk. 1. Homogenization, separation and viscosity tests. Wis. Agric. Exp. Stn. Bull., 60-71

Mayhew E., Lazo R., Vail W.J., King J. \& Green A.M. (1984) Caracterization of liposomes prepared using a microemulsifier. Biochim. Biophys. Acta 775, 169-174

McCormick V. (1968) Hundred years in dairy industry. Dairy Farmers of Canada, D.O.L.C.O. Canada, 76
Muir D.D. \& Banks W. (1986a) Technical note : multiple homogenization of cream liqueurs. J. Food Technol. 21, 229-232

Muir D.D. \& Banks W. (1986b) Cream liqueurs. Hannah Res. 83-88

Siliciano A.A. (1985) Topical liposomes. An update and review of uses and production methods. Cosmet. Toiletries 100, 43

Walstra P. \& Jenness R. (1984) Dairy Chemistry and Physics. John Wiley \& Sons Inc., Canada 